\title{
Vias de acesso transperitoneal e retroperitoneal em cirurgia de aorta: resultados comparativos a longo prazo
}

\author{
Transperitoneal and retroperitoneal approaches to the abdominal aorta: \\ comparison of long-term results
}

\author{
O rlando Caetano Jr. ${ }^{1}$, Kleber Sene Silva ${ }^{1}$, B onno van Bellen ${ }^{2}$
}

\begin{abstract}
Resumo
O bjetivo: $\mathrm{A}$ via de acesso mais utilizada à aorta abdominal para correção da doença obstrutiva ou aneurismática é a transperitoneal, sendo que a retroperitoneal é muitas vezes apontada como causando menos complicaçōes. 0 objetivo deste estudo é comparar as duas vias no tocante às complicações tardias.

M étodo: Para comparação da evolução tardia, foram estudados 96 pacientes retrospectivamente, sendo 81 portadores de aneurisma $e$ 15 de doença aorto-ilíaca. A via retroperitoneal foi usada em 43 pacientes, e a transperitoneal, em 53.

Resultados: Não houve diferença entre as ocorrências de hérnia incisional, flacidez de parede abdominal e ejaculação retrógrada.

Conclusões: $\mathrm{N}$ ão foram observadas diferenças entre os dois tipos de acesso quanto aos parâmetros considerados na avaliação tardia.

Palavras-chave: aorta abdominal, vias de acesso vascular, cirurgia.

Desde que a reconstrução da aorta abdominal, tanto por doença aneurismática ou oclusiva, tornou-se um procedimento de rotina, a via de acesso mais utilizada tem sido a transperitoneal, através da incisão xifopúbica mediana, embora as primeiras abordagens à aorta tenham sido realizadas por via retroperitoneal por Leriche, 0 udot e Dubost ${ }^{1,2}$. 0 acesso foi inicialmente realizado em 1796 por Abernethy e primeiramente descrito em 1808 por $\mathrm{C}_{\text {ooper}}{ }^{2}$. A abordagem à cavidade peritoneal nessa época que antecede as modernas técnicas de anestesia e de controle da infecção cirúrgica era motivo de grande morbi-mortalidade, sendo esta a motivação para o uso da incisão retroperitoneal.
\end{abstract}

1. Ex-estagiário, Serviço de Cirurgia Vascular e Angiologia, Hospital São Joaquim da Real e Benemérita Associação Portuguesa de Beneficência de São Paulo, SP.

2. Chefe do Serviço de Cirurgia Vascular e Angiologia, Hospital São Joaquim da Real e Benemérita Associação Portuguesa de Beneficência de São Paulo, SP. Livre-docente, Faculdade de Medicina, Universidade Estadual de Campinas (UNICAMP), Campinas, SP.

Artigo submetido em 28.03.05, aceito em 31.05.05.

Copyright ( $\odot 2005$ by Sociedade Brasileira de Angiologia e Cirurgia Vascular.
Abstract

O bjective: The transperitoneal approach to the abdominal aorta for surgery of either occlusive or aneurysmal disease is undoubtedly the most often used approach, although the retroperitoneal approach is frequently mentioned as being less prone to complications. The aim of this study was to compare both approaches regarding late complications.

M ethod: Late results were retrospectively studied in 96 patients (81 cases of aortic aneurysm and 15 cases of obstructive disease). The retroperitoneal approach was used in 43 cases and the transperitoneal in 53.

Results: There were no significant differences in the occurrence of incisional hernia, abdominal bulging, and retrograde ejaculation.

Conclusions: There were no differences between the two procedures regarding the parameters considered for late assessment.

Key words: abdominal aorta, vascular access ports, surgery.

A pesar da preferência tradicional pela via transperitoneal, 0 interesse pela utilização da via retroperitoneal como rotina foi novamente despertado em 1963, devido à extensa experiência pessoal de $\mathrm{Rob}^{3}$. D esde então, diversos relatos favoráveis têm surgido, mostrando melhora na qualidade do pós-operatório da cirurgia de aorta, além de facilidade técnica para situações com pacientes de alto risco ou obesidade. Entretanto, outros trabal hos não conseguiram mostrar diferenças entre as duas abordagens em vários dos parâmetros estudados ${ }^{4-10}$. Além disso, a nova experiência com a via retroperitoneal causa a impressão de que esta propicia grande número de complicações de parede, principalmente a flacidez causada pela lesão dos nervos intercostais, que participam da inervação da musculatura da parede abdominal, e que pode alcançar valores de até $37 \%^{11}$. O utras complicações freqüentes são as de natureza sexual no homem eas relacionadas com a destruição dos plexos nervosos periaórticos.

0 objetivo deste trabalho é comparar as duas abordagens, no que tange às complicações tardias, 
em uma série de pacientes submetidos à reconstrução da aorta abdominal.

\section{Pacientes e método Casuística}

Foram considerados, retrospectivamente, 96 pacientes operados no período de 1993 a 1997, tendo sido utilizada a via transperitoneal em 53 pacientes, sendo $44 \mathrm{com}$ aneurisma de aorta abdominal (AAA) e nove com doença oclusiva aorto-ilíaca (D O AI) ea via retroperitoneal em 43 pacientes (37 com AAA e seis com DO Al).

Foram operados 10 pacientes do sexo feminino e 43 do sexo masculino pela via transperitoneal e cinco do sexo feminino e 38 do sexo masculino pela via retroperitoneal.

Treze pacientes do sexo feminino tinham AAA e dois, DOAl, enquanto que 68 do sexo masculino tinham AAA e 13, D O Al.

Foram considerados os casos de hérnia incisional, abaulamento abdominal e ejaculação retrógrada pósoperatórios, obtendo-se a última alteração através de anamnese, e as demais pelo exame físico.

$\mathrm{N}$ ão foram incluídos no estudo os pacientes submetidos a outros procedimentos cirúrgicos concomitantes, como simpatectomia, endarterectomia de artéria ilíaca, ou que foram operados por aneurisma isolado de ilíaca comum, iatrogenia, traumas, aneurisma roto, aneurisma tóraco-abdominal e derivações extra-anatômicas. T ambém não foram incluídos na análise os pacientes do sexo masculino que apresentavam disfunção erétil prévia à cirurgia.

Em ambas as séries, não foram considerados os pacientes que evoluíram a óbito, que ocorreram sem diferença estatística significativa nos dois grupos e por causas não atribuíveis às incisões per se.

\section{Vias de acesso}

$\mathrm{N}$ a via transperitoneal, utilizou-se a incisão xifopúbica mediana, padronizada eutilizada no Serviço, no período empregado nesteestudo, como éclassicamente empregada12. O s pacientes, sob anestesia geral, eram posicionados em decúbito dorsal horizontal, sendo realizada uma incisão na linha mediana abdominal, entre a projeção do processo xifóide e da sínfise púbica, com desvio para a esquerda na cicatriz umbilical, interessando a pele eo tecido subcutâneo. A aponeurose era então incisada na linha al ba, igualmenteentre o processo xifóide e a sínfise púbica. 0 peritônio era aberto, e, após um inventário da cavidade abdominal, as vísceras eram rebatidas para a região direita e superior da cavidade abdominal, com exposição da região retroperitoneal. A pós a real ização da reconstrução arterial , o fechamento da incisão era realizado por planos, com o uso de sutura contínua simples de categut simples 3.0 ou 4.0 para o peritônio, pontos separados simples de mononylon 2.0 ou 0 para a aponeurose, alguns pontos separados invertidos de aproximação com categut simples 3.0 para o tecido celular subcutâneo e pontos separados de D onatti com mononylon 3.0 ou 4.0.

Para via de acesso retroperitoneal, utilizou-se a incisão descrita originalmente por $\mathrm{R} \mathrm{ob}^{3}$, sendo real izadas modificações quanto à altura e abordagem do músculo reto abdominal esquerdo. Os pacientes, sob anestesia geral, eram posicionados com o quadril esquerdo em ângulo de $30^{\circ}$ eo tórax em ângulo de $60^{\circ} \mathrm{em}$ relação à mesa cirúrgica, ficando o membro superior esquerdo posicionado em abdução de $90^{\circ}$, lateralmente à cabeça. A mesa é elevada no seu centro, e suas porções superior e inferior são abaixadas, ficando o paciente na forma de um $\mathrm{V}$ invertido (Figura 1 ). A incisão era iniciada na linha mediana, desde aproximadamente 3 $\mathrm{cm}$ abaixo da cicatriz umbilical, em direção do 100 ou 11 을 espaço intercostal, até a linha axilar anterior, podendo ser mais alta, conforme o nível da aorta que devesse ser abordada (Figura 2).

A aponeurose oblíqua externa e as bainhas do músculo reto abdominal esquerdo eram seccionadas, e o músculo reto abdominal retraído paraa direita, sendo seccionado apenas nos casos em que era necessária maior abordagem das artérias ilíacas. Os músculos oblíquo externo e interno eram seccionados, sendo 0 músculo transverso preferencialmente divulsionado. A berta a fascia transversalis, era feito o descolamento do saco peritoneal em todos os sentidos e rebatido para 0 lado direito do paciente. $\mathrm{N}$ as abordagens mais altas à aorta, feitas por tóraco-freno-laparotomia, era necessária a luxação do rim esquerdo. 0 pinçamento ao nível supracelíaco era realizado através de divulsão do pilar diafragmático esquerdo, sendo a aorta abordada na sua porção póstero-lateral.

Após a realização da reconstrução arterial, 0 fechamento da incisão era realizado por planos, com o uso de sutura contínua simples de categut simples 3.0 ou 4.0 para o peritônio, pontos separados simples de mononylon 2.0 para as aponeuroses, com 


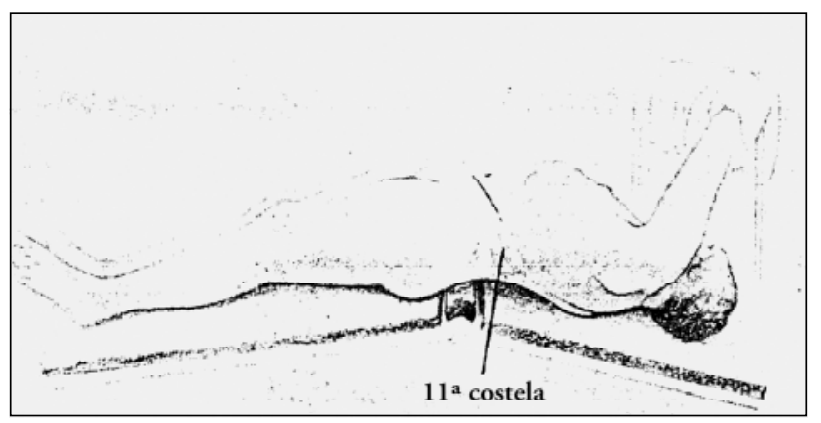

Figura 1 - Posicionamento do paciente

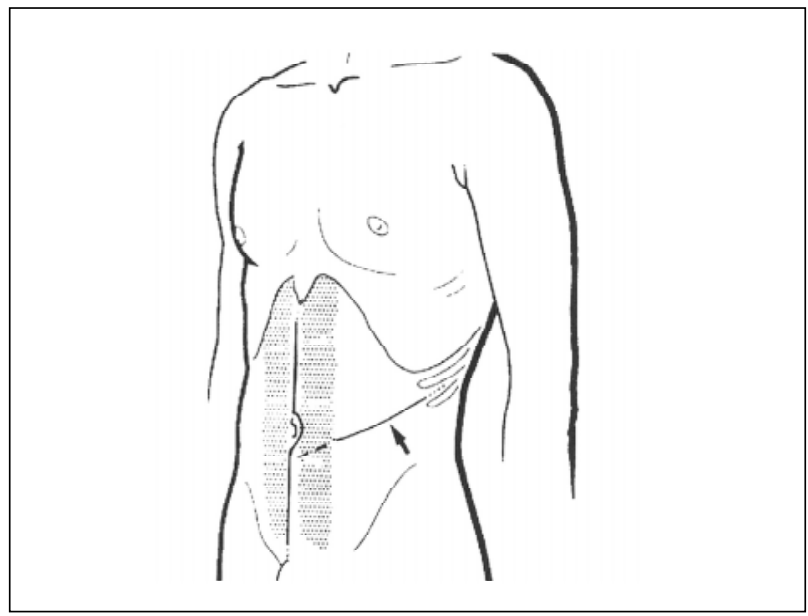

Figura 2 - Incisão retroperitoneal

aproximação dos músculos com pontos separados de categut simples 3.0, al guns pontos separados invertidos de aproximação com categut simples 3.0 para 0 tecido celular subcutâneo e pontos separados de D onatti com mononylon 3.0 ou 4.0.

Para comparação dos resultados obtidos, foi utilizado o teste exato deFisher no nível designificância de 0,05 .

\section{Resultados}

Q uanto à evolução tardia, ocorreu hérnia de parede abdominal em cinco casos, tanto nos casos submetidos a acesso retroperitoneal $(12,2 \%)$ quanto a acesso transperitoneal $(11,6 \%)$, o que também foi semelhante do ponto devista estatístico (T abela 1). A flacidez abdominal ocorreu em três pacientes submetidos a acesso retroperitoneal $(7,3 \%)$ e em igual número de pacientes submetidos a acesso transperitoneal $(6,9 \%)$. A ejaculação retrógrada foi acusada por quatro pacientes subme- tidos a acesso retroperitoneal $(9,7 \%)$ e por seis submetidos a acesso transperitoneal $(14,9 \%)$, diferença sem significância estatística ( $P>0,05)$.

Tabela 1 - Complicações tardias

\begin{tabular}{lcc}
\hline Tipos de acesso & RP $(\mathbf{n}=\mathbf{4 1})$ & TP $(\mathbf{n}=\mathbf{4 3})$ \\
\hline H érnia & $5 \operatorname{casos}(12 \%)$ & $5 \operatorname{casos}(12 \%)$ \\
Flacidez & $3 \operatorname{casos}(7 \%)$ & $3 \cos 0 \mathrm{~s}(7 \%)$ \\
Ejaculação retrógrada & $4 \operatorname{casos}(10 \%)$ & $6 \operatorname{casos}(14 \%)$
\end{tabular}

$\mathrm{RP}=$ retroperitoneal $; \mathrm{TP}=$ transperitoneal. $P>0,05$.

\section{D iscussão}

E mbora seja o acesso maisutilizado na reconstrução da aorta abdominal, a via transperitoneal xifopúbica mediana apresenta al guns problemas. A experiência demonstrada na literatura com a incisão retroperitoneal, no entanto, demonstrou que também essa via apresenta problemas, princi pal mente a longo prazo. No presente trabalho, foi comparada a via retroperitoneal com a via clássica, visando à determinação da eventual superioridade de uma técnica em relação à outra no período pós-operatório tardio de uma série de pacientes.

U ma das importantes complicações tardias é a ejaculação retrógrada, já que se parte do pressuposto que na abordagem retroperitoneal à esquerda haveria maior manipulação de interconexões nervosas na aorta abdominal.

$\mathrm{H}$ istoricamente, a cirurgia vascular no setor aortoilíaco apresenta al to risco de disfunção sexual. $M$ ay et al . relatam $21 \%$ de disfunção erétil pós-aneurismectomia e 34\% pós-reconstrução por D 0 Al ${ }^{13}$. H allböök relata, em uma série pequena, $13 \%$ de impotência e $32 \%$ de ejaculação retrógrada ${ }^{14}$. Várias séries publicadas na década de 1970 demonstraram uma média de 43\% de pacientes com ejaculação retrógrada após cirurgia de revascularização aorto-ilíaca ${ }^{15}$. Flanigan et al. propuseram que a disfunção sexual pós-operatória poderia ser secundária à redução do fluxo sanguíneo pélvico, bem como ser causada pela dissecção defibras autônomas ao redor da aorta abdominal, ao nível da artéria mesentérica inferior e da bifurcação da artéria ilíaca comum esquerda, o que resultaria em perda da potência ou ejaculação retrógrada ${ }^{15,16}$. Em apoio a essa hipótese, 
já se sabe que distúrbios da ejaculação são observados em outros procedimentos, como simpatectomia extensa ${ }^{17}$ e na linfadenectomia retroperitoneal ${ }^{18}$. As alterações da ereção são, por conseguinte, fundamentalmente de causa hemodinâmica, enquanto que as alterações de ejaculação se devem a alterações da inervação pélvica19,20

As abundantes interconexões entre 0 sistema simpático (que regula primariamente a ejaculação) e 0 parassimpático (que regula primariamente a ereção) na região para-aórtica abdominal e plexo hipogástrico, bem como sua importância na função sexual masculina têm sido demonstradas há décadas ${ }^{21}$. A preservação dessesplexosnervosos duranteo procedimento vascular através da abordagem daaorta abdominal maisà direita, associado à dissecção mínima no setor da artéria mesentérica inferior eartéria ilíaca comum esquerda, proporcionaria a diminuição da incidência pós-operatória de disfunção sexual, sem aumento do tempo intra-operatório ${ }^{22}$. U ma técnica inadequada de preservação dos plexos nervosos durante a dissecção aorto-ilíaca pode acarretar incidência de disfunção sexual em elevado percentual dos $\operatorname{casos}^{23}$. H á relatos nos quais não houve preservação nervosa, com incidência de $43 \%$ de ejaculação retrógrada ${ }^{15}$ e $25 \%$ de disfunção erétil iatrogêni$c a^{15,16}$. Com os cuidados adequados, a ejaculação re trógrada foi reduzida para $3 \% 15$.

N o Serviço, é usada de rotina a dissecção mais à direita da aorta abdominal e a preservação dos plexos nervosos encontrados próximos à aorta abdominal. A dissecção da face lateral direita da aorta preserva a inervação simpática relacionada com a atividade sexual do paciente do sexo masculino, principalmente no que diz respeito à ejaculação, a qual pode se tornar seca ou retrógrada pel o comprometimento do esfíncter vesical, que normalmente se contrai no momento da ejaculação, impedindo que o esperma retroceda para a bexiga. A inervação simpática responsável por esse mecanismo compõese dos plexos intermesentérico e do plexo hipogástrico superior, além dos gânglios simpáticos para-aórticos. 0 primeiro consiste em uma rede que recobre a face anterior da aorta, e o segundo, na união dasfibras do plexo intermesentérico em um feixeque se localiza entre as duas artérias ilíacas comuns. As duas formações estão em íntimo contato com os gânglios simpáticos para-aórticos. Os achados retrospectivos encontrados em pacientes que apresentaram a complicação ejaculação retrógrada mostra complicação global de $12 \%$ dos pacientes (Tabela 1), sendo de $10 \%$ no acesso retroperitoneal e 14\% no acesso transperitoneal. Essa diferença não foi estatisticamente significativa. O s dados nospermitem concluir quea ejaculação retrógrada não está relacionada com o tipo de acesso utilizado, mas com a habilidade do cirurgião em minimizar as lesões do plexo nervoso à esquerda da aorta abdominal, na artéria mesentérica inferior e artéria ilíaca esquerda. Sublinha-se, então, a dissecção pela face lateral direita da aorta, o rebater dostecidos(ao invés deseccioná-los), o controleda artéria mesentérica inferior pelo seu óstio, quando possível, a preservação da capa do aneurisma aórtico ou ilíaco com a inserção da prótese no seu interior, a dissecção sempre longitudinal e, preferencialmente, somente a dissecção do mínimo necessário para a realização das anastomoses, especialmente nas artérias ilíacas ${ }^{16}$.

Em relação às complicações relacionadas à parede abdominal (hérnia incisional e flacidez abdominal), os achados da literatura demonstram quea morbidadenão é estatisticamente significante nos dois tipos de acesso vascular6,9, mas há relatos de maior incidência de flacidez na via retroperitoneal ${ }^{10} .0$ relato de $\mathrm{N}$ akajima et al. mostra incidência similar dehérnia eflacidez, mas 0 acesso retroperitoneal utilizado foi mediano ${ }^{8}$. Foram encontrados valores, em relação aos dois tipos de complicação abdominal (hérnia eflacidez abdominal), estatisticamentenão significativos, o que nos permiteargumentar que as incisões retro e transperitoneal apresentam aproximadamente a mesma morbidade em relação às complicações de parede abdominal.

D iabetes mellitus, hipercolesterolemia, hipertensão, obesidade, desnutrição e até mesmo as condições técnicas da sutura abdominal utilizada pelo cirurgião devem ser encaradas como fatores potenciais para 0 desenvolvimento das complicações acima, independente do tipo de incisão utilizada. Algumas complicações, como a flacidez abdominal, podem, como demonstraum estudo anatômico, ser evitadas pelo cuidado com a parte técnica, mormente com a dissecção dos nervos intercostais ${ }^{24}$.

Com relação aos aspectos técnicos, são descritos na literatura vários tipos de acesso retroperitoneal ${ }^{25,26}$, podendo ser divididos em incisões que são uma extensão de inguinotomia 27,28 , transversa ${ }^{29}$, paramediana ${ }^{30,31}$ e mediana $8,32,33$. A incisão pelos flancos pode ser dividida em ântero-lateral, usada

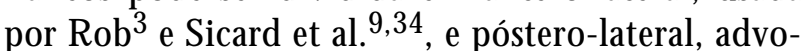
gada por Leather et al. ${ }^{35}$, Shepard et al. ${ }^{36}$ eW illiams et al. ${ }^{37}$. Apesar das diferenças, elas têm suficiente 
similaridade para serem comparadas em conjunto com a incisão xifo-púbica mediana.

\section{Conclusão}

$\mathrm{N}$ ão existevantagem de qualquer incisão quando são consideradas as complicações tardias.

\section{Referências}

1. D ubost $\mathrm{C}, \mathrm{Allary} \mathrm{M}, \mathrm{O}$ economos $\mathrm{N}$. Resection of an aneurysm of the abdominal aorta. Arch Surg. 1952;64:405-8.

2. Shumacker Jr HB. Extraperitoneal approach for vascular operations: retrospective review. South M ed J. 1982;75: 1499-507.

3. RobC. Extraperitoneal approach to theabdominal aorta. Surgery. 1963;53:87-9.

4. Arko FR, Bohannon M M , Lee SD. Retroperitoneal approach for aortic surgery: is it worth it? Cardiovasc Surg. 2001;9:20-6.

5. Ballard JI, Yonemoto $\mathrm{H}$, Killeen JD. Cost-effective aortic exposure: a retroperitoneal experience. Ann Vasc Surg. 2000;14:1-5.

6. C ambriaR P, Brewster D C , Abbott W M . T ransperitoneal versus retroperitoneal approach for aortic reconstruction: arandomized prospective study. J V asc Surg. 1990;11:314-25.

7. Lacroix $\mathrm{H}$, van $\mathrm{H}$ emelrijk J, N evelsteen $\mathrm{A}$. T ransperitoneal versus extraperitoneal approach for routine vascular reconstruction of the abdominal aorta. Acta Chir Belg. 1994;94:1-6.

8. N akajima T, Kawazoe K, Komoda K. M idline retroperitoneal versus midline transperitoneal approach for abdominal aortic aneurysm repair. J V asc Surg. 2000;32:219-23.

9. Sicard GA, Reilly JM, Rubin, BG. Transabdominal versus retroperitoneal incision for abdominal aortic surgery: report of a prospective randomized trial. J V asc Surg. 1995;21:174-83.

10. Sieunarine K, Lawrence-Brown MM, Goodman MA. Comparison of transperitoneal and retroperitoneal approaches for infrarenal aortic surgery: early and late results. J C ardiovasc Surg. 1997;5:71-6.

11. H onig M P, M ason RA, G iron F. W ound complications of the retroperitoneal approach to the aorta and iliac vessels. J V asc Surg 1992; 15: 28-34.

12. Haimovici H. Metodos de exposición de las arterias - El abdomen. In: $\mathrm{H}$ aimovici $\mathrm{H}$, editor. Cirurgia vascular: principios y tecnicas. Barcelona, Salvat Ed, SA.; 1986. p. 224-32.

13. M ay AG, D eweese JA, Rob CG. Changes in sexual function following operation on the abdominal aorta. Surgery. 1969;65:41-7.

14. Hallböök T, Holmquist B. Sexual disturbances following dissection of theaortaand thecommon iliac arteries. J C ardiovasc Surg. 1970;11:255-60.

15. Flanigan DP, Schuler JJ, Keifer T. Elimination of iatrogenic impotence and improvement of sexual function after aortoiliac revascularization. Arch Surg. 1982;117:544-50.

16. Flanigan DP. Postoperative sexual dysfunction following aortoiliac revascularization. In: Rutherford RB, editor. Vascular surgery. Philadelphia: W. B. Saunders C 0., 1995. p. 634-40.

17. Whitelaw GP, Smithwick RH. Some secondary effects of sympathectomy. N Engl M ed J. 1951;245:121-30.

18. Leiter $E$, Brendler $H$. Loss of ejaculation following bilateral retroperitoneal lynphadenectomy. J U rol. 1967;98:375-8.
19. Albers $M T$, Puech-L eão $P, M$ anasterski J. Alteracõos da função sexual masculina após derivações aorto-femorais. Rev H osp Clin Fac M ed S Paulo. 1981;36:167-71.

20. Queral LA, Whitehouse Jr WM, Flinn WR. Pelvic hemodynamics after aortoiliac reconstruction. Surgery. 1979;36:799-809.

21. Langley JN, Anderson H K. The constituents of the hypogastric nerves. J Physiol. 1984;17:177.

22. Schaik J, Baalen JM, Visser MJ. N erve-preserving aortoiliac reconstruction surgery: anatomical study and surgical approach. J V asc Surg. 2001;33:983-9.

23. D epalma RG, Levine SB, Feldman S. Preservation of erectile function after aortoiliac reconstruction. Arch Surg. 1978;113: 958-62.

24. Gardner GP, JosephsLG, RoscaM . Theretroperitoneal incision: an evaluation of postoperative flank "bulge". Arch Surg. 1994;129:753-6.

25. M oreira RC. Acessos extraperitoneais à aorta abdominal: anatomia, técnicas e indicações. Rev Angiol Cirur Vasc. 1996;5:53-62.

26. Araújo AP. Acesso retroperitoneal na cirurgia de aorta. Rev Angiol Cirur Vasc. 1994;3:58-61.

27. Bell DD, M ax RG, M orris H J. Retroperitoneal exposure of the terminal aorta and iliac arteries (thePeter $M$ artin approach). Am J Surg. 1979;138:254-6.

28. Rosengarten $D S, K$ night $B, M$ artin $P$. An approach for operations on the iliac arteries. Br J Surg. 1971;58:365-6.

29. M etz $P, M$ athiesen $F R$. Retroperitoneal approach for implantation of aorto-iliac and aorto-femoral vascular prosthesis. Acta Chir Scand. 1978;144:471-3.

30. H elsby R, M oosa AR. Aorto-iliac reconstruction with special reference to the extraperitoneal approach. $\mathrm{Br} J$ Surg. 1975;62:596-600.

31. T aheri SA, G awronski S, Smith D. Paramedian retroperitoneal approach to the abdominal aorta. J Cardiovasc Surg. 1983;24:529-31.

32. Endo M, Kouichirou K, T subota M. Advantages of using the midline incision right retroperitoneal approach for abdominal aortic aneurysm repair. Jpn J Surg. 1996;26:1-4.

33. Shumacker Jr HB. M idline extraperitoneal exposure of the abdominal aorta and iliac arteries. Surg Gynecol O bstet. 1972;135:791-2.

34. Sicard GA, Freeman MB, Vanderwoude JC. Comparison between the transabdominal and retroperitoneal approach for reconstruction of the infra-renal abdominal aorta. J V asc Surg. 1987;5:19-27.

35. Leather RP, Shah D M, Kaufman IL. Comparative analysis of retroperitoneal and transperitoneal aortic replacement for aneurysm. Surg Gynecol O bstet. 1989;168:387-93.

36. Shepard AD , Scott GR, M ackey W C. Retroperitoneal approach to high-risk abdominal aneurysm. Arch Surg. 1986;121:444-9.

37. W illiamsGM , RicottaJ, Zinner M . Theextended retroperitoneal approach for treatment of extensive atherosclerosis of the aorta and renal vessels. Surgery. 1980;88:846-55.

\section{Correspondência:}

O rlando C aetano J r.

Rua Banco das Palmas, 124

CEP 02016-020 - São Paulo, SP

Tel./Fax: (11) 6977.0711

E-mail: orlandocaetano@terra.com.br 